\title{
Thermal re-emission model
}

\author{
Amit K. Chattopadhyay* \\ Department of Chemistry, University of Virginia, McCormick Road, Charlottesville, Virginia 22904 \\ and COMP/Laboratory of Physics, Helsinki University of Technology, P.O. Box 1100, FIN-02015 HUT, Finland
}

(Received 1 March 2001; Revised manuscript received 16 August 2001; published 4 January 2002)

\begin{abstract}
Starting from a continuum description, we study the nonequilibrium roughening of a thermal re-emission model for etching in one and two spatial dimensions. Using standard analytical techniques, we map our problem to a generalized version of an earlier nonlocal KPZ (Kardar-Parisi-Zhang) model. In $2+1$ dimensions, the values of the roughness and the dynamic exponents calculated from our theory go like $\alpha \approx z \approx 1$ and in $1+1$ dimensions, the exponents resemble the KPZ values for low vapor pressure, supporting experimental results. Interestingly, Galilean invariance is maintained throughout.
\end{abstract}

DOI: 10.1103/PhysRevB.65.041405

PACS number(s): 68.35.Ct, 05.40.-a, 05.70.Ln, 64.60.Ht

The subject of kinetic roughening and nonequilibrium growths, has been the center interest of far-from-equilibrium physics for more than two decades now. This is mainly due to two reasons: on the one hand, due to the ongoing revolution in the world of microphysics in recent years, the demand of the age is to understand and implement the underlying mechanism associated. ${ }^{1}$ On the other hand, they seem to correlate fields even as diverse as ecological growths, propagation of a crack-front, stock-market predictions, etc. ${ }^{2}$ Although the processes which have been probed so far, have mostly been concerned only with local effects, such as molecular-beam-epitaxy (MBE) growth, conventional diffusive growths, etc., the importance of the nonlocal effects, have been known as early as the 1950's. ${ }^{3}$ Later on, with the advent of more sophisticated experimental techniques, nonlinear effects involving physical vapor deposition (PVC),${ }^{1,4-6}$ sputtering techniques and associated growth and etching of plasma fonts have assumed a position of paramount importance. Whereas in standard MBE type of growths, the vapor atoms are targeted in a direction normal to the substrate, so that growth is decided by the local environment only, in case of shadowing growths by sputter deposition, vapor atoms are incident at random angles to the surface, so that nonlocal factors gain prominence in this case. $^{7-11}$ There have been several experimental follow-ups of this sputtering mechanism as well. ${ }^{12-14}$

The concept of shadowing effect in a sputtering growth (or etching) essentially arrived with the observation that thin films often exhibit "an extended network of grooves and voids in their interiors" 11 giving rise to columnar structures. The basic idea is the following. Since in a sputtering growth (etching), particles are allowed to be deposited (deroded) on the surface from all possible angles at random, the rate of growth is taken to be proportional to the exposure angle $\theta(x)$, which is a function of the position of incidence of the incoming particle. Now, as the hills have greater exposure area, they receive more atoms than the valleys. Thus the hills continue to grow steeper compared to the depleted valleys, which naturally gives rise to an instability in the system. The idea has been very ingeniously, but intelligently related to the growth of the relatively larger stalks, in a grassy lawn, which suppress the growth of the shorter ones ${ }^{11}$ and in the process giving rise to a rough contour.
In the theoretical front, this phenomenon of shadowing growth (decay), or its partner, the thermal re-emission instability has inspired a series of works in $1+1$ dimensions ${ }^{7,8,11,15-17}$ and in $2+1$ dimensions. ${ }^{14,18,19}$ The theoretical forays in fact started with the paper by Karunasiri et $\mathrm{al}^{7}{ }^{7}$ where from a direct numerical integration of the dynamical equation, they were able to show that the selfsimilarity of the contour, evident at small values of the diffusion constant, is modified by the growth of flat films, beyond a critical height, as the value of the diffusion constant is increased. Taking clues from their arguments, Roland and $\mathrm{Guo}^{15}$ went on to calculate the value of the roughness constant, in 1+1 dimensions (albeit in the context of a shadowing model) and further predicted that in the low temperature phase, the system resembles a KPZ universality class (in agreement with Karunasiri et al.). ${ }^{7}$ This concept of nonlocal, shadowing effect was later modified, ${ }^{9,11}$ where a net nonlocal flux was observed to give rise to the inherent columnar structures found in experiments. Later on, the domain of $2+1$ dimension was also probed with the advent of advanced numerical integration algorithms and Monte-Carlo simulations. ${ }^{18,19}$ However, all these attempts, both in $1+1$ and $2+1$ dimensions, being predominantly numerical, either through direct numerical integration of a fundamental Langevin-type equation, or through Monte-Carlo simulation, and all the more, giving contradictory values of the exponents obtained by different groups, we ventured an analytical derivation to have a final say regarding the universality class of these type of sputtered mechanisms. In the process, we will see that our findings correlate the available experimental and numerical observations (of one of these groups) in $2+1$ dimensions and predicts scaling in $1+1$ dimensions, too.

With the assumption that the shadowing effect provides the dominant instability in the system, we apply the nonlocal model proposed by Zhao et al. ${ }^{14,18,19}$ The model is given by

$$
\frac{\partial h(\vec{r}, t)}{\partial t}=\nu \nabla^{2} h(\vec{r}, t) \pm \sqrt{1+(\vec{\nabla} h)^{2}} R(\vec{r}, t)+\eta(\vec{r}, t)
$$

and

$$
\left\langle\eta(\vec{r}, t) \eta\left(\vec{r}^{\prime}, t^{\prime}\right)\right\rangle=2 D \delta^{2}\left(\vec{r}-\vec{r}^{\prime}\right) \delta\left(t-t^{\prime}\right)
$$



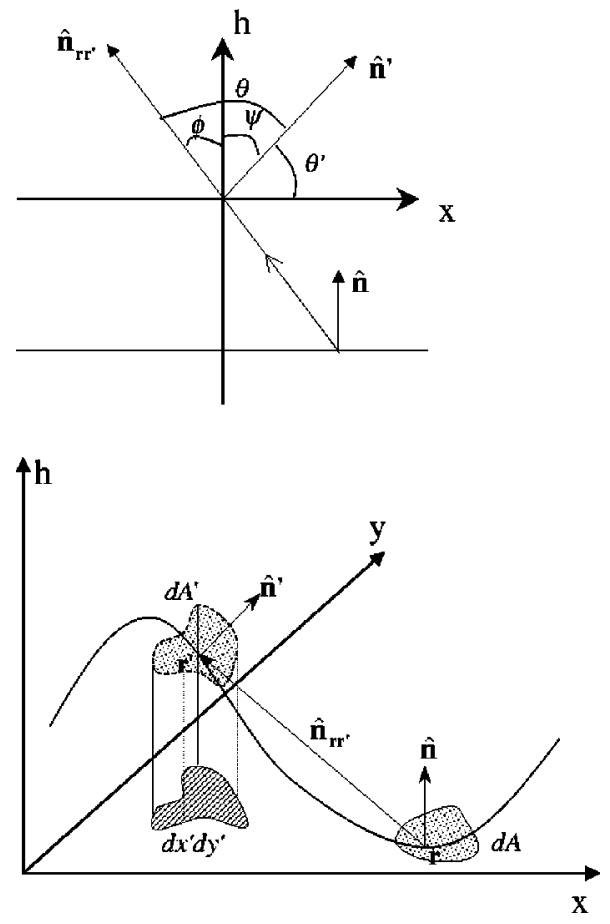

FIG. 1. Relative orientations of the unit normals at $\vec{r}$ and $\vec{r}^{\prime}$ and the co-ordinate system described by them.

where the first term on the right hand side of Eq. (1) provides the diffusive relaxing mechanism for the growing (or etching) surface and the last term signifies the collective effect of randomness in the system, taken to be a Gaussian noise. The middle term is the nonlocal, nonlinear term detailing the effects of thermal re-emission and is given by

$$
R(\vec{r}, t)=s_{0} F_{0}(\vec{r}, t)+s_{1} F_{1}(\vec{r}, t),
$$

where $s_{0}$ is the zeroth order sticking coefficient and $s_{1}$ is generated due to the re-emission mechanism. ${ }^{14}$ Here we consider first-order thermal re-emission, that is neglect the effects of $s_{i}(i>1)$. Plugging again from the same reference and applying the same logic, we consider the flux of the $m$ th order particle at position $\vec{r}$ as $F_{m}(\vec{r}, t)$ which is given by

$$
\begin{aligned}
F_{m+1}(\vec{r}, t)= & \left(1-s_{m}\right) \int Z\left(\vec{r}, \vec{r}^{\prime}, t\right) F_{m}\left(\vec{r}^{\prime}, t\right) \\
& \times \frac{\left(\hat{n} \overrightarrow{r_{r}^{\prime}} \cdot \hat{n}\right) P\left(\hat{n}_{\vec{r}^{\prime}}, \hat{n}^{\prime}\right)}{\left(\vec{r}-\vec{r}^{\prime}\right)^{2}+\left(h-h^{\prime}\right)^{2}} d A^{\prime} .
\end{aligned}
$$

For our case of first-order re-emission, we are concerned with $m=0$ and 1 . Here $\hat{n}$ is the unit normal to the surface at $\vec{r}$, pointing outwards, $\hat{n}^{\prime}$ is the unit normal at $\vec{r}^{\prime}$ and $\hat{n}_{\vec{r} r^{\prime}}$ is the unit vector connecting $\vec{r}$ and $\vec{r}^{\prime}$ (see Fig. 1). $P\left(\hat{n}_{\vec{r}^{\prime}}, \hat{n}^{\prime}\right)$ is the probability, per unit solid angle, that the reemitted particle flies off along $\hat{n}_{\vec{r}, \vec{r}}$ and is expressed as $\left(\hat{n}_{\vec{r}^{\prime}} \cdot \hat{n}^{\prime}\right) / \pi \cdot{ }^{18} Z\left(\vec{r}, \vec{r}^{\prime}, t\right)$ is equal to unity except when there is no line of sight between the surface elements at $\vec{r}$ and $\vec{r}^{\prime}$ and zero otherwise. The nonlinear factor $\sqrt{1+(\vec{\nabla} h)^{2}}$ which is multiplied with $R(\vec{r}, t)$, signifies the lateral growth (or etching, as the case may be) associated and the "+" and "- " signs as its prefix, refer to growth or etching, respectively. In the following analysis, we will consider parameter values as in Ref. 19 (that is we will be dealing with etching due to sputtering). Thus, for our case, $F_{0}=4, s_{0} \approx 0$, and $s_{1} \approx 1$. Also $P\left(\hat{n}_{r^{\prime}} \vec{r}, \hat{n}^{\prime}\right)=\left(\hat{n}_{\vec{r}^{\prime}} \cdot \hat{n}^{\prime}\right) / \pi$, assuming thermally re-emitted flux, although this is more of a simplification ${ }^{20}$ than exact truth. With the above description of the complete equation, we proceed to determine the dynamics in the $2+1$ dimensional case. Later on, we will discuss our results with reference to $1+1$ dimensions, as well.

Combining Eqs. (1), (3), and (4) and taking $\psi$ as the angle between $\vec{r}$ and $\vec{r}^{\prime}$ (see Fig. 1), the dynamical etching equation reduces to

$$
\frac{\partial h}{\partial t} \approx \nu \nabla^{2} h-\left[1+\frac{1}{2}(\vec{\nabla} h)^{2}\right] F_{1}(\vec{r}, t)+\eta(\vec{r}, t),
$$

where

$$
\begin{aligned}
F_{1}(\vec{r}, t) \approx & \iint \frac{4 \cos \theta}{\pi} \frac{\sin \left[\theta+\theta^{\prime}\right]}{\left(\vec{r}-\vec{r}^{\prime}\right)^{2}+\left(h-h^{\prime}\right)^{2}} \\
& \times \sqrt{1+\left[\vec{\nabla} h^{\prime}\left(\vec{r}^{\prime}, \theta^{\prime}\right)\right]^{2}} r^{\prime} d r^{\prime} d \theta^{\prime}
\end{aligned}
$$

where $\theta=$ angle between $\hat{n}_{\vec{r} r^{\prime}}$ and $\hat{n}^{\prime}=\phi+\psi$ as in Fig. 1 and $\theta^{\prime}$ is again defined as in Fig. 1. In arriving at Eqs. (5) and (6), we have deliberately chosen $\hat{n}$ as one of the axes in the two-dimensional plane, to simplify calculations. This can be done, since on the average this holds true. Also the standard lateral growth assumption, $|\vec{\nabla} h|<1$ has been employed. This $F_{1}(\vec{r}, t)$ can be further reduced to

$$
F_{1}(\vec{r}, t) \approx \frac{8\langle\cos \theta\rangle^{2}}{\pi} \int_{-L}^{L} d r^{\prime} \frac{\left|r^{\prime}-r\right|\left[1+\frac{1}{2}\left(\partial_{r^{\prime}} h^{\prime}\right)^{2}\right]}{\left(r^{\prime}-r\right)^{2}+\left(h-h^{\prime}\right)^{2}},
$$

where $L$ is the size of the system. It is important to mention here that in deriving Eq. (7) from Eq. (6), we have used the mean-value theorem, since $\pi / 2-\delta<\theta^{\prime}<\pi / 2+\delta$ ( $\delta$ is an angular strip around $h$ ), the range being evident from Fig. 1 . The " $\approx$ " sign justifies the fact that we have taken a meanvalued average, represented by " \langle\rangle " around the $h$-axis, thereby removing $\langle\cos \theta\rangle$ outside the integral as a first-order approximation. Simplifying further, we arrive at the analytically tractable form of $F_{1}(\vec{r}, t)$, as given below:

$$
F_{1}(\vec{r}, t) \approx \frac{8\langle\cos \theta\rangle^{2}}{\pi} \int_{-L}^{L} d r^{\prime} \frac{\left[1-\frac{1}{2}\left(\partial_{r^{\prime}} h^{\prime}\right)^{2}\right]}{\left|r^{\prime}-r\right|} .
$$

In arriving at the above equations, we have put on a very standard assumption for any nonlocal model that the height difference $\left(h-h^{\prime}\right)$, calculated between any two points $\vec{r}$ and $\vec{r}^{\prime}$ of the growing surface should be much smaller than their distance of separation, i.e., $\left|h-h^{\prime}\right| \ll\left|\vec{r}-\vec{r}^{\prime}\right|$, a basic property expected of any nonlocal process. 
With this assumption and the mean-valued average done beforehand, the equation of motion now becomes

$$
\begin{aligned}
\frac{\partial h}{\partial t} \approx & \nu \nabla^{2} h-\frac{8\langle\cos \theta\rangle^{2}}{\pi} \int_{-L}^{L} d r^{\prime} \frac{1}{\left|r^{\prime}-r\right|}\left[1+\frac{1}{2}(\vec{\nabla} h)^{2}\right] \\
& +\frac{4\langle\cos \theta\rangle^{2}}{\pi} \int_{-L}^{L} d r^{\prime} \frac{1}{\left|r^{\prime}-r\right|}\left[\partial_{r^{\prime}} h\left(r^{\prime}\right)\right]^{2}+\eta(\vec{r}, t) .
\end{aligned}
$$

Now, we try to look at the possible large-time, long-distance behavior of the system. We can easily see that the KPZ part, ${ }^{21}$ constituting the second term on the right-hand side of the above equation will vanish as the system size is taken to be sufficiently large. In deriving the above form, terms higher than $(\vec{\nabla} h)^{2}$ order have been neglected. The final equation now looks like

$$
\frac{\partial h}{\partial t} \approx \nu \nabla^{2} h+\int_{0}^{L} d r^{\prime} \phi\left(r, r^{\prime}\right)\left|\partial_{r^{\prime}} h\right|^{2}+\eta(\vec{r}, t),
$$

where

$$
\phi\left(r, r^{\prime}\right)=\frac{4 \lambda \pi}{\left|r^{\prime}-r\right|},
$$

$\lambda=\lambda_{0}\langle\cos \theta\rangle^{2}$ is an adjustable coupling parameter, such that we will later put $\lambda_{0}$ equal to unity. The fact that the assumptions employed above are perfectly trustworthy, can be cross checked from the fact that Eq. (11) maintains translational invariance which was an important feature of our starting Eq. (4).

Equation (10) can be easily mapped to the phenomenological equation considered in Ref. 22. The only trick lies in a suitable wave-vector representation of the effective longrange potential $\phi\left(r, r^{\prime}\right)$ in our case. Obviously, this cannot be a simple plug-in from the earlier equation of motion, ${ }^{22}$ since, the interacting potential is apparently a multivalued function here. To progress further, we move on to the wavevector representation of this interacting potential which is given by the scaled relation

$$
\phi\left(k, k^{\prime}\right)=4 \frac{\lambda}{k} f\left(\frac{k}{k^{\prime}}\right) .
$$

Here the scaling function looks like

$$
f\left(\frac{k}{k^{\prime}}\right)=\int d X X e^{-i X} \int \frac{Y e^{-i Y}}{\left.Y-\frac{k}{k^{\prime}} X\right)} .
$$

Considering the scaling ansatz

$$
f\left(k, k^{\prime}\right)=f\left(\frac{k}{k^{\prime}}\right)=A\left(\frac{k}{k^{\prime}}\right)^{\eta}
$$

we get

$$
\phi\left(k, k^{\prime}\right) \approx \lambda \frac{k^{\eta-1}}{k^{\prime \eta}}
$$

and our job now is to evaluate the definite scaling behavior for $f\left(k, k^{\prime}\right)$ by the evaluation of a number for $\eta$ from Eq. (13). ${ }^{23}$ Applying simple Laplace transform and going through the standard steps, it is easy to see that the dominating contribution of the double integral in Eq. (13) implies that $\eta=1$ (Ref. 24) and this gives the value

$$
\phi\left(k, k^{\prime}\right) \approx \lambda \frac{1}{k^{\prime}},
$$

i.e., the major contributing part of the potential is effectively reduced to a single variable mode. Now, we can simply plug-in results from Ref. 22 and write down the dynamic exponent $z$ as

$$
z=2+K
$$

where

$$
K=-24 / 23=-1.04
$$

for our case. ${ }^{23}$ One obvious point to be noted here is the fact that owing to the Galilean invariance of Eq. (9), we can easily see that

$$
\alpha+z=2
$$

and interestingly enough, the general tendency of the system is to flow towards a short-ranged fixed point (the long-ranged fixed point comes out to be unphysical with the specific parameter values, for our particular case). This effect, as we will see, also holds sway in $1+1$ dimensions, where the system flows towards the KPZ fixed point.

Combining the last two equations, we get

$$
\alpha=-K \text {. }
$$

Thus the critical exponents come out as

$$
\begin{aligned}
& z=\frac{22}{23}=0.96, \\
& \alpha=\frac{24}{23}=1.04, \\
& \beta=\frac{\alpha}{z} \approx 1.08,
\end{aligned}
$$

i.e., $\alpha \approx \beta \approx z \approx 1$ in reasonable agreement with experimental and numerical findings ${ }^{14,18,19}$ (experimental values are: $\alpha$ $=0.96 \pm 0.06, \beta=0.91 \pm 0.03$, and $z=1.05 \pm 0.08$ ), within experimental error bars. The fact that the theory (and also experiment ${ }^{18}$ ) predicts $\alpha \approx 1$ indicates that the effects of overhangs might be marginal (Ref. 1, p. 110). Also to be noted is the invariance of the Galilean identity $\alpha+z=2$. Before concluding this portion, it must be mentioned that for the opposite scenario, i.e., growth under first-order thermal re-emission, an identical analysis as above shows immediately that now the reduced dynamical equation has a form 
nearly the same as that in Eq. (10) but with a negative nonlocal potential. This automatically suggests that due to the attractive nature of this potential, the growth finally stops at sufficiently large times ("smoothens") and $\beta \approx 0 .{ }^{18}$ Interestingly, we find that even without thermal re-emission, this marked change in the scaling properties, depending on whether it is a growth or an etching process has been discussed elsewhere also. $^{25}$

For the $1+1$ dimensional case, we follow exactly similar lines, the only modification being the consideration of $\theta^{\prime}$ $=0$ and $\theta=0$ or $\pi$ (depending on growth or decay, respectively) in Eq. (6). Thereafter, proceeding likewise, the dominating long-ranged part comes out to be $v(r) \int_{0}^{L} d r^{\prime}\left(\partial_{r^{\prime}} h\right)^{2}$, with $v(r) \approx L / r$. Thus in the large time limit, as $r \rightarrow L$, we see that the system approaches the conventional KPZ fixed point and naturally the exponents also resemble the KPZ universality class, which can be looked upon as sort of an analogy with the shadowing case. ${ }^{7}$ To avoid unnecessary repetition of identical calculations, as in the 2+1-dimensional case, we have neglected any further details in $1+1$ dimensions.

All said and done, however, there is still one open question which needs to be resolved. This is the fact that in spite of both the available short-ranged and long-ranged fixed points in the 2+1-dimensional case, the system chooses the short-ranged fixed point (an alternative statement that there is Galilean invariance in the system, since the other fixed point basically gives an unphysical picture with $\alpha<0$ ), although the shadowing effect fundamentally remains a nonlocal contribution. This seems to suggest that whenever we are talking about nonlocal interactions, it does not necessarily mean that the long-ranged structure should control the associated dynamics. Instead the short-ranged part of the contribution might also take the upper hand, though obviously depending on the type of interaction we are considering. The issue seems to demand further studies. As an adjoinder, we would like to mention that in the 1+1-dimensional situation, being basically dominated by the KPZ fixed point, no such complexity arises over there.

The author (A.K.C.) would like to sincerely acknowledge illuminating interactions with Y.-P. Zhao and J. Drotar. All discussions with Dr. Sergei Egorov and Y.-J. Lee are acknowledged. A.K.C. is indebted to M.J. Alava for his hospitality at the HUT.
*Present address: Max-Planck-Institute for the Physics of Complex Systems, Nöthnitzer Str. 38, Dresden, D-01187, Germany. Email address: akc@mpipks-dresden.mpg.de

${ }^{1}$ A. L. Barabasi and H. E. Stanley, in Fractal Concepts in Surface Growth (Cambridge University Press, Cambridge 1994); F. Family and T. Vicsek, in Dynamics of Fractal Surfaces (World Scientific, Singapore, 1991).

${ }^{2}$ D. Sornette, Physica A 290, 211 (2001); H. J. Blok, Ph.D. thesis, cond-mat/0010211; E. Canessa, Eur. Phys. J. B 20, 591 (2001); V. Aji and N. Goldenfeld, Phys. Rev. Lett. 86, 1007 (2001).

${ }^{3}$ H. Konig and T. Helwig, Optik (Stuttgart) 6, 111 (1950).

${ }^{4}$ R. J. Hoekstra, M. J. Kusher, V. Sukharev, and P. Schoenborn, J. Vac. Sci. Technol. B 16, 2102 (1998).

${ }^{5}$ V. K. Singh, E. S. G. Shaqfeh, and J. P. McVittie, J. Vac. Sci. Technol. B 12, 2952 (1994); ibid. 10, 1091 (1992).

${ }^{6}$ R. Petri, P. Brault, O. Vatel, D. Henry, E. Andre, P. Dumas, and F. Sulvan, J. Appl. Phys. 75(11), 7498 (1994).

${ }^{7}$ R. P. U. Karunasiri, R. Bruinsma, and J. Rudnick, Phys. Rev. Lett. 62, 788 (1989).

${ }^{8}$ R. P. U. Karunasiri, R. Bruinsma, and J. Rudnick, Phys. Rev. Lett. 63, 693 (1989).

${ }^{9}$ G. S. Bales and A. Zangwill, Phys. Rev. Lett. 63, 692 (1989).

${ }^{10}$ C. Tang, S. Alexander, and R. Bruinsma, Phys. Rev. Lett. 64, 772 (1990).

${ }^{11}$ G. S. Bales, R. Bruinsma, E. A. Eklund, R. P. U. Karunasiri, J. Rudnick, and Z. Zangwill, Science 249, 264 (1990).

${ }^{12}$ H. You, R. P. Chiarello, H. K. Kim, and K. G. Vandervoort, Phys.
Rev. Lett. 70, 2900 (1993).

${ }^{13}$ D. Le Bellac, G. A. Niklasson, and C. G. Granqvist, Europhys. Lett. 32, 155 (1995).

${ }^{14}$ Y.-P. Zhao, J. T. Drotar, G.-C. Wang, and T.-M. Lu, Phys. Rev. Lett. 82, 4882 (1999).

${ }^{15}$ C. Roland and H. Guo, Phys. Rev. Lett. 66, 2104 (1991).

${ }^{16}$ J. H. Yao, C. Roland, and H. Guo, Phys. Rev. A 45, 3903 (1992).

${ }^{17}$ J. Krug and P. Meakin, Phys. Rev. E 47, R17 (1993).

${ }^{18}$ J. T. Drotar, Y.-P. Zhao, T.-M. Lu, and G.-C. Wang, Phys. Rev. B 61, 3012 (2000).

${ }^{19}$ J. T. Drotar, Y.-P. Zhao, T.-M. Lu, and G.-C. Wang, Phys. Rev. B 62, 2118 (2000).

${ }^{20}$ G. S. Hwang, C. M. Anderson, M. J. Gordon, T. A. Moore, T. K. Minton, and K. P. Giapis, Phys. Rev. Lett. 77, 3049 (1996).

${ }^{21}$ M. Kardar, G. Parisi, and Y. C. Zhang, Phys. Rev. Lett. 56, 889 (1996).

${ }^{22}$ S. Mukherji and S. M. Bhattacharjee, Phys. Rev. Lett. 79, 2502 (1997).

${ }^{23}$ We are putting $d=1$ and $\rho=1$ in the results of Ref. 22. Here it should be remembered that although we are still in the twodimensional plane, the structure of our nonlocal part features a one-dimensional integration only. Hence $d=1$.

${ }^{24}$ From simple power counts from both sides of the self-energy equation, $\eta=1$, since otherwise the right side of the equation becomes a function of two variables, as opposed to the left side, which is only dependent on the external momentum.

${ }^{25}$ J. Krug and P. Meakin, Phys. Rev. Lett. 66, 703 (1991). 